Original Research Paper

\title{
A Randomized Population Constructive Heuristic for the Team Orienteering Problem
}

\author{
Manal Zettam \\ Informatics, Systems and Optimization Laboratory, \\ Department of computer Science, Faculty of Science, Ibn Tofail University Kenitra, Morocco
}

Article history

Received: 18-12-2016

Revised: $12-06-2017$

Accepted: 16-06-2017

Email: zettammanal@gmail.com

\begin{abstract}
The NP-hard (complete) team orienteering problem is a particular vehicle routing problem with the aim of maximizing the profits gained from visiting control points without exceeding a travel cost limit. The team orienteering problem has a number of applications in several fields such as athlete recruiting, technician routing and tourist trip. Therefore, solving optimally the team orienteering problem would play a major role in logistic management. In this study, a novel randomized population constructive heuristic is introduced. This heuristic constructs a diversified initial population for population-based metaheuristics. The heuristics proved its efficiency. Indeed, experiments conducted on the well-known benchmarks of the team orienteering problem show that the initial population constructed by the presented heuristic wraps the best-known solution for 131 benchmarks and good solutions for a great number of benchmarks.
\end{abstract}

Keywords: Orienteering Problem, Team Orienteering Problem, PopulationBased Meta-Heuristic

\section{Introduction}

The Orienteering Problem (OP) was first introduced by (Golden et al., 1987). It originates from orienteering sport. According to (Zettam and Elbenani, 2016) the Orienteering sport is defined as an outdoor sport, played in heavily forested and mountainous areas. A set of control points are located in the forest. Each control point is associated with a score. Assuming that the start control point and the end control point are fixed. Competitors equipped with compass and map are required to visit a subset of control points starting from the start control point and ending at the end control point with the aim of maximizing their total score within a predefined amount of time. Several variants of OP has been described in literature such as The Team Orienteering Problem, the Orienteering Problem with Time Windows, the team orienteering problem with time windows and the time-dependent orienteering Problem. OP and its variant were given a great interest as results of their applications (Vansteenwegen et al., 2011). Selective travelling salesperson problem (Tsiligirides, 1984), home fuel delivery problem (Golden et al., 1987), single-ring design problem (Thomadsen and Stidsen, 2003) and mobile tourist guide problem (Souffriau et al., 2008) are some of well known application of OP and its variants. For more details readers are referred to (Vansteenwegen et al., 2011).

The team orienteering problem TOP extends OP. It was first introduced by (Chao et al., 1996). Competitors are subdivided into teams. The competitors of a given team collaborate to maximize the score within a predefined amount of time or distance limit. Each control point is visited once by a member of a given team. Even though two exact methods have been proposed to solve the team orienteering problem, it still be considered as an NP-hard (complete) problem according to (Chao et al., 1996). Therefore, a number of heuristics and metaheuristics have been developed to solve TOP. Lin (2013) proposed a multi-start simulated annealing algorithm that combines the simulated annealing algorithm and the multi-start hill climbing algorithm. Butt and Cavalier (1994) presented a mixed integer programming solved by a greedy method that adds the best pair of vertices to the solution tours at each iteration. They claims that their approach do well with relatively small number of control points within a tour. In the approach proposed by (Chao et al., 1996), the initial solution is constructed by inserting Control points into paths using the cheapest insertion rule. If unsigned control points remain new paths are constructed. The constructed initial solution is improved by using 1-point movement, 2-point exchange 
and 2-opt operator with record-to-record framework. Tang and Miller-Hook (2005) proposed a Tabu search embedded with adoptive memory. This method generates better solution with more computational time compared to (Chao et al., 1996) for a number of instances. (Archetti et al., 2007) addressed a variable neighborhood approach which outperforms (Tang and Miller-Hooks, 2005; Chao et al., 1996) approaches. Ke et al. (2008) proposed an Ant Colony Approach that embedded one of the three following methods: Sequential deterministic-concurrent method, randomconcurrent method and simultaneous method. The performance of the Ant Colony Approach with the sequential method is comparable to (Archetti et al., 2007). Vansteenwegen et al. (2009) introduced the disturb method which combines a guided local search method and a diversifying mechanism. The disturb method find the best-known solutions in shorter computational time compared to the existing methods. (Bouly et al., 2009) proposed a PSO-inspired algorithm which updated the best-known solution for one instance use this style when you need to begin a new paragraph.

In this study, we propose a randomized population constructive heuristic for the Team orienteering problem. This heuristic constructs a diversified initial population for population-based metaheuristics. It constructs solutions based on randomly generated permutations of control points. The solutions within the initial population are diversified with different costs and tour lengths. A number of permutations are randomly generated. For each permutation of control points a given number of path beginning from the starting control point and ending at the arrival control point are constructed. The best solution of the population is then enhanced by a local search.

The rest of this paper is organized as follows. The first section addresses the team orienteering problem. The first section also introduces the mathematical model adopted in this study. The second section details the proposed heuristic. The fourth section involves the computational results followed by concluding remarks and proposal for future works in the fifth section.

\section{Mathematical Formulation}

Given a set of $n$ control points and $m$ competitors. The control points are usually named locations and competitors are usually named tours in the literature of TOP. The main goal of TOP is to construct $m$ tours starting from the departure (location 1) and ending at the arrival (location $n$ ) that maximize the total score denoted $s$. A travel time or length of a tour cannot exceed $T_{\max }$. In the present paper, we employ the Euclidean distance to calculate the length tours. Table
1 shows an example of TOP instances where $T_{\max }=$ $20, m=2$. Table 1 contains locations, $X Y$ coordinates and scores. The solution of the instance shown in Table 1 is represented in Fig. 1.

In this study, a mathematical model similar to the one used by Lin (2013) is described as follows:

$\operatorname{Maximize} \sum_{k=1}^{m} \sum_{i=2}^{n-1} S_{i} y_{i k}$

Subject to:

$\sum_{k=1}^{m} \sum_{j=2}^{n-1} x_{1 j k}=\sum_{k=1}^{m} \sum_{i=2}^{n-1} x_{i n k}=m$

$\sum_{i=1}^{n-1} x_{i l k}=\sum_{j=2}^{n} x_{l j k}=y_{l p}, \forall l=2, \ldots, n-1 ; \forall k=1, \ldots, m$

$\sum_{k=1}^{m} y_{i k} \leq 1, \forall i=2, \ldots, n-1$

$\sum_{i=1}^{n-1} \sum_{j=2}^{n} t_{i j} x_{i j k} \leq T_{\max }, \forall k=1, \ldots, m$

$\sum_{\substack{i, j, U \\ i<j}} x_{i j k} \leq|U|-1 \quad(\mathrm{U} \subset \mathrm{V} \backslash\{1, n\}: 2 \leq|U| \leq \mathrm{n}-2 ; k=1, \ldots, m)$

$x_{i j k}, y_{i k} \in\{0,1\}, \forall \mathrm{i}, \mathrm{j}=1, \ldots, \mathrm{n} ; \forall k=1, \ldots, m$

Where:

$S_{i} \quad=\quad$ Is the score associated to the $i$ th location

$t_{i j}=$ The length of the path starting at the $i$ th location and ending at the $j$ th location. The travel length cannot exceed $T_{\max }$

$V \quad=\quad$ Is the set of locations

$U \quad=$ Is a subset of $V$

$x_{i j m}=1=$ if, in tour $m$, a visit to location is followed by a visit to location, 0 otherwise

$y_{i k}=1=$ if location is $i$ visited on tour $k, 0$ otherwise

$s_{i k}=$ The start time of the service at location $i$ on tour $k$

The objective function to maximize is represented by Equation 1. The constraint that all tours starts from location 1 and ends at location $n$ is ensured by Equation 2. The connectivity of tours is maintained by Equation 3 . Constraint (4) guarantees that every location is visited at most once. Constraint (5) guarantees that length limitation constraint is not violated for each tour. Constraint (6) excludes sub-tours. Constraint (7) states that the variables $x$ and $y$ are binary. 
Manal Zettam / Journal of Computer Science 2017, 13 (9): 440.451 DOI: $10.3844 /$ jessp.2017.440.451

Table 1. An example of TOP instances

\begin{tabular}{|c|c|c|c|}
\hline Location & $\mathrm{X}$ & $\mathrm{Y}$ & $\mathrm{S}$ \\
\hline 1 & 10.500 & 14.400 & 0 \\
\hline 2 & 18.000 & 15.900 & 10 \\
\hline 3 & 18.300 & 13.300 & 10 \\
\hline 4 & 16.500 & 9.300 & 10 \\
\hline 5 & 15.400 & 11.000 & 10 \\
\hline 6 & 14.900 & 13.200 & 5 \\
\hline 7 & 16.300 & 13.300 & 5 \\
\hline 8 & 16.400 & 17.800 & 5 \\
\hline 9 & 15.000 & 17.900 & 5 \\
\hline 10 & 16.100 & 19.600 & 10 \\
\hline 11 & 15.700 & 20.600 & 10 \\
\hline 12 & 13.200 & 20.100 & 10 \\
\hline 13 & 14.300 & 15.300 & 5 \\
\hline 14 & 14.000 & 5.100 & 10 \\
\hline 15 & 11.400 & 6.700 & 15 \\
\hline 16 & 8.300 & 5.000 & 15 \\
\hline 17 & 7.900 & 9.800 & 10 \\
\hline 18 & 11.400 & 12.000 & 5 \\
\hline 19 & 11.200 & 17.600 & 5 \\
\hline 20 & 10.100 & 18.700 & 5 \\
\hline 21 & 11.700 & 20.300 & 10 \\
\hline 22 & 10.200 & 22.100 & 10 \\
\hline 23 & 9.700 & 23.800 & 10 \\
\hline 24 & 10.100 & 26.400 & 15 \\
\hline 25 & 7.400 & 24.000 & 15 \\
\hline 26 & 8.200 & 19.900 & 15 \\
\hline 27 & 8.700 & 17.700 & 10 \\
\hline 28 & 8.900 & 13.600 & 10 \\
\hline 29 & 5.600 & 11.100 & 10 \\
\hline 30 & 4.900 & 18.900 & 10 \\
\hline 31 & 7.300 & 18.800 & 10 \\
\hline 32 & 11.200 & 14.100 & 0 \\
\hline
\end{tabular}

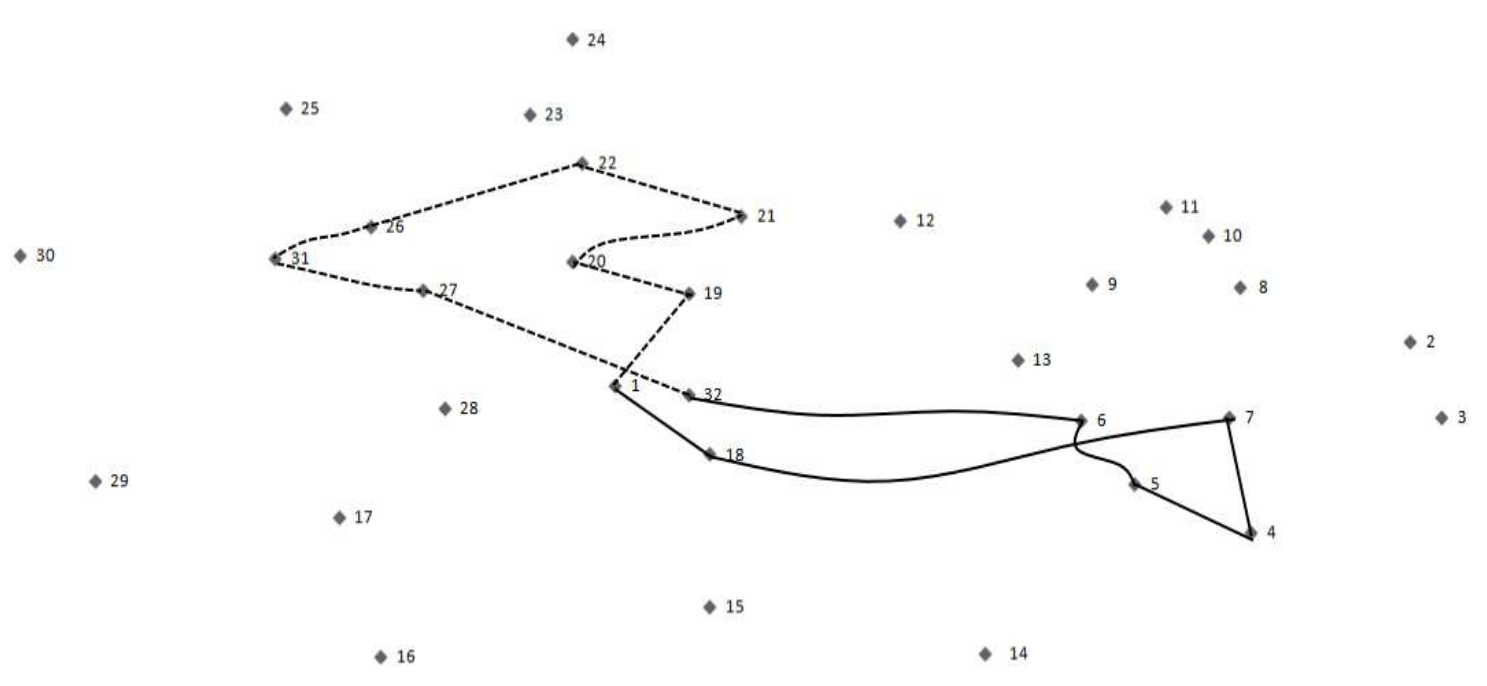

Fig. 1. A solution example

\section{The Novel Randomized Heuristic}

The heuristic randomly generates a predefined number of permutations. Solutions are then constructed on the basis of the generated permutations. Each solution is represented by $m$ connected tours. Given a permutation of $n$ elements. Each element represents a location. For each tour $k$, the heuristic seeks a permutation. If the $i$ th location is not contained in other tours and if adding the $i$ th location does not violate fifth constraint described in the mathematical model, the $i$ th location is added to the tour $k$ th. Otherwise, the 
heuristic goes to the next iteration and so forth. Finally the best solution of the population is enhanced via a local search. The local search used in this study employs the swap and the add/drop operators. The solution is enhanced 10 times with a neighbourhood size equal to the half of the number of customer in the first tour. Our heuristic is describes in details in the algorithm (1) below. $\operatorname{dist}(i, j)$ denotes the Euclidean distance between two location $i$ and $j$.

Algorithm (1): The novel randomize heuristic

Inputs: The number of tours denoted $m$,

The maximum length of a tour $T_{\max }$,

The set of locations $V=\{1,2, \ldots, n\}$,

The size of the population $l$.

Start

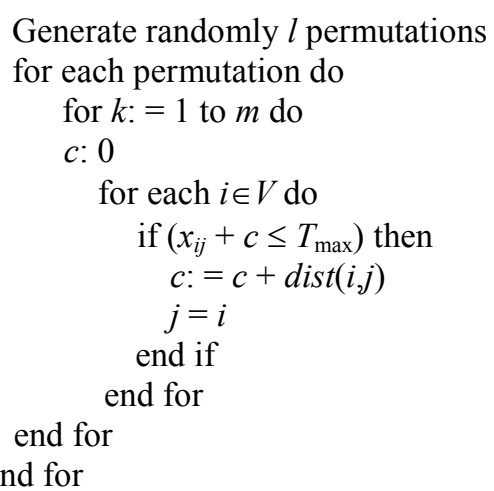

Apply the local search to enhance the best solution.

Output: population of feasible solutions

The randomized heuristic reaches best-known solutions for 131 of benchmarks in a minor computational time. The randomized heuristic also generates average solutions for the majority of the reminder benchmarks. A good initial population would allow population based metaheuristics to find good solutions in an optimistic computational time with less computational efforts.

\section{Results and Discussion}

The proposed heuristic was implemented using JAVA language and was run on a PC with an Intel Core i5-2540 M 2.60 GHz processor. To evaluate the performance of the proposed heuristic, each benchmark of TOP was tested over ten trial and compared to the best-known solution. The benchmarks we used in this study are available at (https://www.hds.utc.fr/ moukrim). Table 2 summarizes the characteristics of problem benchmarks sets. The first number of a problem indicates the set number, e.g., p2.2, p2.3 and p2.4 belong to set 2 . The coordinates and score of each location are identical for the instances belonging to a given problem set. The second number in the problem set indicates the number of tours, e.g., p2.3 means that there are 3 tours.

We ran our heuristic 20 times on each instance in order to select the best obtained solution. Table 3 to 23 contain the problem nomination, the best-known solution in the literature and also the best solution in the population we obtained by applying the proposed randomized heuristic. The third and fifth column of each table contain the results obtained by generating a population of $10 \times T_{\max }$ and 10 elements. The best-known solutions in bold are obtained by exacts methods. In this section, we use the best-known solutions provided by (Kim et al., 2013).

Table 2. Benchmarks characteristics

\begin{tabular}{llll}
\hline Problem set & Number of Locations & Number of sub-problem & $T_{\max }$ \\
\hline p1.2 & 32 & 18 & $2.5-42.5$ \\
p1.3 & 32 & 18 & $1.7-28.3$ \\
p1.4 & 32 & 18 & $1.2-21.2$ \\
p2.2 & 21 & 11 & $7.5-22.5$ \\
p2.3 & 21 & 11 & $5.0-15.0$ \\
p2.4 & 21 & 11 & $3.8-11.2$ \\
p3.2 & 33 & 20 & $5.0-36.7$ \\
p3.3 & 33 & 20 & $3.8-27.5$ \\
p3.4 & 33 & 20 & $25.0-120.0$ \\
p4.2 & 100 & 20 & $16.7-80.0$ \\
p4.3 & 100 & 20 & $12.5-60.0$ \\
p4.4 & 100 & 20 & $2.5-65.0$ \\
p5.2 & 66 & 26 & $1.7-43.3$ \\
p5.3 & 66 & 26 & $1.2-32.5$ \\
p5.4 & 66 & 26 & $7.5-40.0$ \\
p6.2 & 64 & 14 & $1.2-32.5$ \\
p6.3 & 64 & 14 & $5.0-26.7$ \\
p6.4 & 64 & 14 & $3.8-20.0$ \\
p7.2 & 102 & 20 & $10.0-200.0$ \\
p7.3 & 102 & 20 & $6.7-133.3$ \\
p7.4 & 102 & 20 & $5.0-100.0$ \\
\hline
\end{tabular}


Manal Zettam / Journal of Computer Science 2017, 13 (9): 440.451 DOI: $10.3844 /$ jessp.2017.440.451

Table 3. The computational results for data set 1 with $\mathrm{m}=2$

\begin{tabular}{lllllll} 
Problem & Best-known solution & Best-obtained solution & Time (Second) & Best-obtained solution & Time (Second) & $T_{\max }$ \\
\hline p1.2.a & 0 & 0 & 0.016 & 0 & 0.006 & 2.5 \\
p1.2.b & 15 & 15 & 0.013 & 15 & 0.008 & 5.0 \\
p1.2.c & 20 & 20 & 0.016 & 20 & 0.006 & 7.5 \\
p1.2.d & 30 & 30 & 0.012 & 30 & 0.007 & 10.0 \\
p1.2.e & 45 & 45 & 0.021 & 40 & 0.017 & 12.5 \\
p1.2.f & 80 & 80 & 0.065 & 60 & 0.008 & 15.0 \\
p1.2.g & 90 & 85 & 0.074 & 65 & 0.009 & 17.5 \\
p1.2.h & 110 & 100 & 0.093 & 75 & 0.008 & 20.0 \\
p1.2.i & 135 & 120 & 0.124 & 90 & 0.016 & 23.0 \\
p1.2.j & 155 & 130 & 0.112 & 120 & 0.011 & 25.0 \\
p1.2.k & 175 & 140 & 0.228 & 110 & 0.011 & 27.5 \\
p1.2.1 & 195 & 155 & 0.134 & 155 & 0.012 & 30.0 \\
p1.2.m & 215 & 165 & 0.148 & 120 & 0.016 & 32.5 \\
p1.2.n & 235 & 165 & 0.172 & 125 & 0.02 & 35.0 \\
p1.2.0 & 240 & 170 & 0.167 & 120 & 0.019 & 36.5 \\
p1.2.p & 250 & 180 & 0.169 & 130 & 0.013 & 37.5 \\
p1.2.q & 265 & 180 & 0.181 & 130 & 0.013 & 40.0 \\
p1.2.r & 280 & 195 & 0.21 & 145 & 0.023 & 42.5 \\
\hline
\end{tabular}

Table 4. The computational results for data set 1 with $\mathrm{m}=3$

\begin{tabular}{lllllll} 
Problem & Best-known solution & Best-obtained solution & Time (Second) & Best-obtained solution & Time (Second) & $T_{\max }$ \\
\hline p1.3.a & 0 & 0 & 0.013 & 0 & 0.007 & 1.7 \\
p1.3.b & 0 & 0 & 0.024 & 0 & 0.008 & 3.3 \\
p1.3.c & 15 & 15 & 0.025 & 15 & 0.01 & 5.0 \\
p1.3.d & 15 & 15 & 0.044 & 15 & 0.009 & 6.7 \\
p1.3.e & 30 & 30 & 0.03 & 30 & 0.01 & 8.3 \\
p1.3.f & 40 & 40 & 0.055 & 40 & 0.05 & 10.0 \\
p1.3.g & 50 & 50 & 0.054 & 50 & 0.01 & 11.7 \\
p1.3.h & 70 & 70 & 0.074 & 65 & 0.01 & 13.3 \\
p1.3.i & 105 & 100 & 0.091 & 80 & 0.01 & 15.3 \\
p1.3.j & 115 & 105 & 0.108 & 90 & 0.03 & 16.7 \\
p1.3.k & 135 & 120 & 0.022 & 105 & 0.06 & 18.3 \\
p1.3.1 & 155 & 130 & 0.024 & 105 & 0.01 & 20.0 \\
p1.3.m & 175 & 140 & 0.023 & 120 & 0.04 & 21.7 \\
p1.3.n & 190 & 160 & 0.025 & 135 & 0.01 & 23.3 \\
p1.3.o & 205 & 165 & 0.021 & 145 & 0.01 & 24.3 \\
p1.3.p & 220 & 170 & 0.012 & 155 & 0.02 & 25.0 \\
p1.3.q & 230 & 180 & 0.035 & 150 & 0.02 & 26.7 \\
p1.3.r & 250 & 185 & 0.028 & 165 & 0.01 & 28.3 \\
\hline
\end{tabular}

Table 5. The computational results for data set 1 with $\mathrm{m}=4$

\begin{tabular}{lllllll}
\hline Problem & Best-known solution & Best-obtained solution & Time (Second) & Best-obtained solution & Time (Second) & $T_{\max }$ \\
\hline p1.4.a & 0 & 0 & 0.006 & 0 & 0.004 & 1.2 \\
p1.4.b & 0 & 0 & 0.006 & 0 & 0.005 & 2.5 \\
p1.4.c & 0 & 0 & 0.006 & 0 & 0.004 & 3.8 \\
p1.4.d & 15 & 15 & 0.007 & 15 & 0.008 & 5.0 \\
p1.4.e & 15 & 15 & 0.009 & 15 & 0.007 & 6.2 \\
p1.4.f & 25 & 25 & 0.009 & 25 & 0.006 & 7.5 \\
p1.4.g & 35 & 35 & 0.011 & 35 & 0.005 & 8.8 \\
p1.4.h & 45 & 45 & 0.013 & 45 & 0.007 & 10.0 \\
p1.4.i & 60 & 60 & 0.015 & 55 & 0.006 & 11.5 \\
p1.4.j & 75 & 75 & 0.015 & 70 & 0.007 & 12.5 \\
p1.4.k & 100 & 100 & 0.017 & 85 & 0.007 & 13.8 \\
p1.4.1 & 120 & 120 & 0.024 & 110 & 0.008 & 15.0 \\
p1.4.m & 130 & 125 & 0.023 & 110 & 0.008 & 16.2 \\
p1.4.n & 155 & 140 & 0.035 & 120 & 0.012 & 17.5 \\
p1.4.o & 165 & 150 & 0.093 & 130 & 0.013 & 18.2 \\
p1.4.p & 175 & 155 & 0.095 & 130 & 0.01 & 18.8 \\
p1.4.q & 190 & 160 & 0.032 & 125 & 0.013 & 20.0 \\
p1.4.r & 210 & 165 & 0.023 & 145 & 0.019 & 21.2 \\
\hline
\end{tabular}


Manal Zettam / Journal of Computer Science 2017, 13 (9): 440.451

DOI: $10.3844 /$ jessp.2017.440.451

Table 6 . The computational results for data set 2 with $\mathrm{m}=2$

\begin{tabular}{lllllll}
\hline Problem & Best-known solution & Best-obtained solution & Time (Second) & Best-obtained solution & Time (Second) & $T_{\max }$ \\
\hline p2.2.a & 90 & 90 & 0.03 & 85 & 0.005 & 7.5 \\
p2.2.b & 120 & 120 & 0.009 & 120 & 0.005 & 10.0 \\
p2.2.c & 140 & 140 & 0.011 & 130 & 0.018 & 11.5 \\
p2.2.d & 160 & 160 & 0.048 & 145 & 0.012 & 12.5 \\
p2.2.e & 190 & 180 & 0.011 & 145 & 0.008 & 13.5 \\
p2.2.f & 200 & 200 & 0.013 & 180 & 0.009 & 15.0 \\
p2.2.g & 200 & 200 & 0.042 & 185 & 0.008 & 16.0 \\
p2.2.h & 230 & 230 & 0.065 & 185 & 0.009 & 17.5 \\
p2.2.i & 230 & 230 & 0.012 & 230 & 0.013 & 19.0 \\
p2.2.j & 260 & 230 & 0.023 & 210 & 0.011 & 20.0 \\
p2.2.k & 275 & 260 & 0.099 & 230 & 0.025 & 22.5 \\
\hline
\end{tabular}

Table 7. The computational results for data set 2 with $\mathrm{m}=3$

\begin{tabular}{|c|c|c|c|c|c|c|}
\hline Problem & Best-known solution & Best-obtained solution & Time (Second) & Best-obtained solution & Time (Second) & $T_{\max }$ \\
\hline $\mathrm{p} 2.3 . \mathrm{a}$ & 70 & 70 & 0.028 & 70 & 0.013 & 5.0 \\
\hline $\mathrm{p} 2.3 . \mathrm{b}$ & 70 & 70 & 0.035 & 70 & 0.047 & 6.7 \\
\hline $\mathrm{p} 2.3 . \mathrm{c}$ & 105 & 105 & 0.036 & 105 & 0.007 & 7.7 \\
\hline $\mathrm{p} 2.3 . \mathrm{d}$ & 105 & 105 & 0.039 & 105 & 0.007 & 8.3 \\
\hline $\mathrm{p} 2.3 . \mathrm{e}$ & 120 & 120 & 0.045 & 120 & 0.01 & 9.0 \\
\hline $\mathrm{p} 2.3 . \mathrm{f}$ & 120 & 120 & 0.009 & 120 & 0.004 & 10.0 \\
\hline $\mathrm{p} 2.3 . \mathrm{g}$ & 145 & 145 & 0.01 & 140 & 0.01 & 10.7 \\
\hline $\mathrm{p} 2.3 . \mathrm{h}$ & 165 & 165 & 0.05 & 160 & 0.016 & 11.7 \\
\hline $\mathrm{p} 2.3 . \mathrm{i}$ & 200 & 200 & 0.012 & 180 & 0.019 & 12.7 \\
\hline $\mathrm{p} 2.3 . \mathrm{j}$ & 200 & 200 & 0.017 & 185 & 0.06 & 13.3 \\
\hline $\mathrm{p} 2.3 . \mathrm{k}$ & 200 & 200 & 0.012 & 200 & 0.008 & 15.0 \\
\hline
\end{tabular}

Table 8 . The computational results for data set 2 with $\mathrm{m}=4$

\begin{tabular}{lllllll}
\hline Problem & Best-known solution & Best-obtained solution & Time (Second) & Best-obtained solution & Time (Second) & $T_{\max }$ \\
\hline p2.4.a & 10 & 10 & 0.008 & 10 & 0.005 & 3.8 \\
p2.4.b & 70 & 70 & 0.009 & 70 & 0.006 & 5.0 \\
p2.4.c & 70 & 70 & 0.008 & 70 & 0.006 & 5.8 \\
p2.4.d & 70 & 70 & 0.007 & 70 & 0.006 & 6.2 \\
p2.4.e & 70 & 70 & 0.008 & 70 & 0.008 & 6.8 \\
p2.4.f & 105 & 105 & 0.01 & 105 & 0.019 & 7.5 \\
p2.4.g & 105 & 105 & 0.009 & 105 & 0.008 & 8.0 \\
p2.4.h & 120 & 120 & 0.011 & 120 & 0.009 & 8.8 \\
p2.4.i & 120 & 120 & 0.012 & 120 & 0.007 & 9.5 \\
p2.4.j & 120 & 120 & 0.011 & 120 & 0.013 & 10.0 \\
p2.4.k & 180 & 180 & 0.011 & 165 & 0.009 & 11.2 \\
\hline
\end{tabular}

Table 9 . The computational results for data set 3 with $\mathrm{m}=2$

\begin{tabular}{lllllll}
\hline Problem & Best-known solution & Best-obtained solution & Time (Second) & Best-obtained solution & Time (Second) & $T_{\max }$ \\
\hline p3.2.a & 90 & 90 & 0.006 & 80 & 0.006 & 7.5 \\
p3.2.b & 150 & 150 & 0.01 & 120 & 0.008 & 10.0 \\
p3.2.c & 180 & 180 & 0.014 & 160 & 0.015 & 12.5 \\
p3.2.d & 220 & 190 & 0.014 & 170 & 0.004 & 15.0 \\
p3.2.e & 260 & 230 & 0.018 & 210 & 0.008 & 17.5 \\
p3.2.f & 300 & 240 & 0.019 & 210 & 0.011 & 20.0 \\
p3.2.g & 360 & 270 & 0.023 & 190 & 0.007 & 22.5 \\
p3.2.h & 410 & 300 & 0.024 & 210 & 0.019 & 25.0 \\
p3.2.i & 460 & 330 & 0.028 & 270 & 0.019 & 27.5 \\
p3.2.j & 510 & 350 & 0.065 & 280 & 0.019 & 30.0 \\
p3.2.k & 550 & 370 & 0.028 & 270 & 0.014 & 32.5 \\
p3.2.1 & 590 & 380 & 0.032 & 350 & 0.015 & 35.0 \\
p3.2.m & 620 & 390 & 0.034 & 320 & 0.027 & 37.5 \\
p3.2.n & 660 & 0.055 & 340 & 0.066 & 40.0 \\
p3.2.o & 690 & 420 & 0.031 & 370 & 0.013 & 42.5 \\
p3.2.p & 720 & 430 & 0.036 & 360 & 0.015 & 45.0 \\
p3.2.q & 760 & 440 & 0.076 & 380 & 0.022 & 47.5 \\
p3.2.r & 790 & 460 & 0.043 & 430 & 0.072 & 50.0 \\
p3.2.s & 800 & 510 & 0.045 & 450 & 0.025 & 52.5 \\
p3.2.t & 800 & 520 & 0.048 & 520 & & 55.0 \\
\hline
\end{tabular}


Manal Zettam / Journal of Computer Science 2017, 13 (9): 440.451 DOI: $10.3844 /$ jessp.2017.440.451

\begin{tabular}{|c|c|c|c|c|c|c|}
\hline Problem & Best-known solution & Best-obtained solution & Time (Second) & Best-obtained solution & Time (Second) & $T_{\max }$ \\
\hline p3.3.a & 30 & 30 & 0.008 & 30 & 0.006 & 5.0 \\
\hline p3.3.b & 90 & 90 & 0.011 & 90 & 0.008 & 6.7 \\
\hline p3.3.c & 120 & 120 & 0.012 & 120 & 0.008 & 8.3 \\
\hline p3.3.d & 170 & 170 & 0.027 & 170 & 0.014 & 10.0 \\
\hline p3.3.e & 200 & 200 & 0.014 & 200 & 0.04 & 11.7 \\
\hline p3.3.f & 230 & 220 & 0.018 & 180 & 0.006 & 13.3 \\
\hline p3.3.g & 270 & 240 & 0.032 & 200 & 0.05 & 15.0 \\
\hline p3.3.h & 300 & 270 & 0.018 & 250 & 0.009 & 16.7 \\
\hline p3.3.i & 330 & 300 & 0.021 & 280 & 0.012 & 18.3 \\
\hline p3.3.j & 380 & 310 & 0.043 & 270 & 0.02 & 20.0 \\
\hline $\mathrm{p} 3.3 . \mathrm{k}$ & 440 & 370 & 0.026 & 310 & 0.017 & 21.7 \\
\hline p3.3.1 & 480 & 370 & 0.058 & 320 & 0.023 & 23.3 \\
\hline p3.3.m & 520 & 370 & 0.031 & 320 & 0.024 & 25.0 \\
\hline p3.3.n & 570 & 430 & 0.026 & 330 & 0.013 & 26.7 \\
\hline p3.3.o & 590 & 420 & 0.033 & 380 & 0.014 & 28.3 \\
\hline p3.3.p & 640 & 470 & 0.068 & 370 & 0.014 & 30.0 \\
\hline p3.3.q & 680 & 500 & 0.036 & 480 & 0.014 & 31.7 \\
\hline p3.3.r & 710 & 530 & 0.036 & 460 & 0.019 & 33.3 \\
\hline p3.3.s & 720 & 510 & 0.037 & 460 & 0.015 & 35.0 \\
\hline p3.3.t & 760 & 550 & 0.05 & 550 & 0.04 & 36.7 \\
\hline
\end{tabular}

Table 11. The computational results for data set 3 with $\mathrm{m}=4$

\begin{tabular}{|c|c|c|c|c|c|c|}
\hline Problem & Best-known solution & Best-obtained solution & Time (Second) & Best-obtained solution & Time (Second) & $T_{\max }$ \\
\hline p3.4.a & 20 & 20 & 0.021 & 20 & 0.015 & 3.8 \\
\hline p3.4.b & 30 & 30 & 0.011 & 30 & 0.008 & 5.0 \\
\hline p3.4.c & 90 & 90 & 0.011 & 90 & 0.006 & 6.2 \\
\hline p3.4.d & 100 & 100 & 0.012 & 100 & 0.009 & 7.5 \\
\hline p3.4.e & 140 & 140 & 0.023 & 140 & 0.009 & 8.8 \\
\hline p3.4.f & 190 & 190 & 0.015 & 190 & 0.011 & 10.0 \\
\hline p3.4.g & 220 & 220 & 0.018 & 220 & 0.013 & 11.2 \\
\hline p3.4.h & 240 & 240 & 0.021 & 240 & 0.013 & 12.5 \\
\hline p3.4.i & 270 & 240 & 0.019 & 230 & 0.012 & 13.8 \\
\hline p3.4.j & 310 & 300 & 0.024 & 260 & 0.014 & 15.0 \\
\hline p3.4.k & 350 & 310 & 0.023 & 270 & 0.016 & 16.2 \\
\hline p3.4.1 & 380 & 320 & 0.044 & 310 & 0.015 & 17.5 \\
\hline p3.4.m & 390 & 350 & 0.056 & 290 & 0.013 & 18.8 \\
\hline p3.4.n & 440 & 370 & 0.027 & 330 & 0.012 & 20.0 \\
\hline p3.4.o & 500 & 410 & 0.055 & 380 & 0.02 & 21.2 \\
\hline p3.4.p & 560 & 430 & 0.037 & 390 & 0.025 & 22.5 \\
\hline p3.4.q & 560 & 440 & 0.031 & 380 & 0.017 & 23.8 \\
\hline p3.4.r & 600 & 470 & 0.19 & 410 & 0.016 & 25.0 \\
\hline p3.4.s & 670 & 500 & 0.038 & 430 & 0.026 & 26.2 \\
\hline p3.4.t & 670 & 500 & 0.045 & 410 & 0.013 & 27.5 \\
\hline
\end{tabular}

Table 12. The computational results for data set 4 with $\mathrm{m}=2$

\begin{tabular}{|c|c|c|c|c|c|c|}
\hline Problem & Best-known solution & Best-obtained solution & Time (Second) & Best-obtained solution & Time (Second) & $T_{\max }$ \\
\hline$\overline{\mathrm{p} 4.2 . \mathrm{a}}$ & 206 & 152 & 0.041 & 131 & 0.046 & 25.0 \\
\hline $\mathrm{p} 4.2 . \mathrm{b}$ & 341 & 222 & 0.048 & 152 & 0.011 & 30.0 \\
\hline p4.2.c & 452 & 231 & 0.063 & 169 & 0.011 & 35.0 \\
\hline p4.2.d & 531 & 260 & 0.063 & 180 & 0.012 & 40.0 \\
\hline p4.2.e & 618 & 253 & 0.067 & 200 & 0.017 & 45.0 \\
\hline p4.2.f & 687 & 263 & 0.066 & 193 & 0.041 & 50.0 \\
\hline p4.2.g & 757 & 299 & 0.086 & 216 & 0.027 & 55.0 \\
\hline $\mathrm{p} 4.2 . \mathrm{h}$ & 835 & 316 & 0.09 & 236 & 0.018 & 60.0 \\
\hline p4.2.i & 918 & 310 & 0.093 & 232 & 0.02 & 65.0 \\
\hline $\mathrm{p} 4.2 . \mathrm{j}$ & 965 & 322 & 0.098 & 297 & 0.024 & 70.0 \\
\hline $\mathrm{p} 4.2 . \mathrm{k}$ & 1022 & 356 & 0.108 & 282 & 0.016 & 75.0 \\
\hline p4.2.1 & 1074 & 354 & 0.103 & 296 & 0.021 & 80.0 \\
\hline $\mathrm{p} 4.2 . \mathrm{m}$ & 1132 & 375 & 0.123 & 316 & 0.023 & 85.0 \\
\hline $\mathrm{p} 4.2 . \mathrm{n}$ & 1174 & 421 & 0.13 & 294 & 0.021 & 90.0 \\
\hline p4.2.o & 1218 & 389 & 0.292 & 355 & 0.11 & 95.0 \\
\hline p4.2.p & 1242 & 435 & 0.132 & 315 & 0.019 & 100.0 \\
\hline p4.2.q & 1268 & 435 & 0.142 & 320 & 0.03 & 105.0 \\
\hline $\mathrm{p} 4.2 . \mathrm{r}$ & 1292 & 487 & 0.182 & 318 & 0.02 & 110.0 \\
\hline p4.2.s & 1304 & 483 & 0.151 & 340 & 0.102 & 115.0 \\
\hline p4.2.t & 1306 & 455 & 0.16 & 348 & 0.04 & 120.0 \\
\hline
\end{tabular}


Manal Zettam / Journal of Computer Science 2017, 13 (9): 440.451

DOI: $10.3844 /$ jessp.2017.440.451

Table 13. The computational results for data set 4 with $\mathrm{m}=3$

\begin{tabular}{|c|c|c|c|c|c|c|}
\hline Problem & Best-known solution & Best-obtained solution & Time (Second) & Best-obtained solution & Time (Second) & $T_{\max }$ \\
\hline p4.3.a & 0 & 0 & 0.011 & 0 & 0.002 & 16.7 \\
\hline $\mathrm{p} 4.3 . \mathrm{b}$ & 38 & 38 & 0.04 & 38 & 0.07 & 20.0 \\
\hline p4.3.c & 193 & 173 & 0.05 & 123 & 0.02 & 23.3 \\
\hline p4.3.d & 335 & 210 & 0.051 & 187 & 0.013 & 26.7 \\
\hline p4.3.e & 468 & 255 & 0.06 & 212 & 0.01 & 30.0 \\
\hline p4.3.f & 579 & 292 & 0.065 & 248 & 0.017 & 33.3 \\
\hline p4.3.g & 653 & 298 & 0.071 & 265 & 0.018 & 36.7 \\
\hline p4.3.h & 729 & 328 & 0.086 & 272 & 0.026 & 40.0 \\
\hline $\mathrm{p} 4.3 . \mathrm{i}$ & 809 & 314 & 0.091 & 274 & 0.023 & 43.3 \\
\hline $\mathrm{p} 4.3 . \mathrm{j}$ & 861 & 363 & 0.092 & 310 & 0.014 & 46.7 \\
\hline $\mathrm{p} 4.3 . \mathrm{k}$ & 919 & 353 & 0.251 & 305 & 0.099 & 50.0 \\
\hline p4.3.1 & 979 & 370 & 0.12 & 321 & 0.031 & 53.3 \\
\hline $\mathrm{p} 4.3 . \mathrm{m}$ & 1063 & 412 & 0.126 & 300 & 0.013 & 56.7 \\
\hline p4.3.n & 1121 & 405 & 0.131 & 330 & 0.017 & 60.0 \\
\hline p4.3.o & 1172 & 415 & 0.123 & 342 & 0.039 & 63.3 \\
\hline p4.3.p & 1222 & 425 & 0.16 & 418 & 0.076 & 66.7 \\
\hline p4.3.q & 1253 & 457 & 0.144 & 360 & 0.022 & 70.0 \\
\hline $\mathrm{p} 4.3 . \mathrm{r}$ & 1273 & 451 & 0.153 & 388 & 0.026 & 73.3 \\
\hline $\mathrm{p} 4.3 . \mathrm{s}$ & 1295 & 478 & 0.16 & 371 & 0.024 & 76.7 \\
\hline p4.3.t & 1305 & 475 & 0.165 & 421 & 0.039 & 80.0 \\
\hline
\end{tabular}

Table 14. The computational results for data set 4 with $\mathrm{m}=4$

\begin{tabular}{|c|c|c|c|c|c|c|}
\hline Problem & Best-known solution & Best-obtained solution & Time (Second) & Best-obtained solution & Time (Second) & $T_{\max }$ \\
\hline$\overline{\mathrm{p} 4.4 . \mathrm{a}}$ & 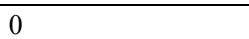 & 0 & 0.034 & 0 & 0.008 & 12.5 \\
\hline $\mathrm{p} 4.4 . \mathrm{b}$ & 0 & 0 & 0.03 & 0 & 0.008 & 15.0 \\
\hline p4.4.c & 0 & 0 & 0.05 & 0 & 0.01 & 17.5 \\
\hline p4.4.d & 38 & 38 & 0.054 & 38 & 0.01 & 20.0 \\
\hline p4.4.e & 183 & 183 & 0.223 & 172 & 0.013 & 22.5 \\
\hline p4.4.f & 324 & 270 & 0.07 & 208 & 0.017 & 25.0 \\
\hline p4.4.g & 461 & 277 & 0.08 & 244 & 0.036 & 27.5 \\
\hline $\mathrm{p} 4.4 . \mathrm{h}$ & 571 & 333 & 0.092 & 275 & 0.021 & 30.0 \\
\hline $\mathrm{p} 4.4 . \mathrm{i}$ & 657 & 332 & 0.088 & 292 & 0.033 & 32.5 \\
\hline $\mathrm{p} 4.4 . \mathrm{j}$ & 732 & 350 & 0.091 & 342 & 0.025 & 35.0 \\
\hline $\mathrm{p} 4.4 . \mathrm{k}$ & 821 & 403 & 0.093 & 379 & 0.022 & 37.5 \\
\hline p4.4.1 & 880 & 417 & 0.103 & 349 & 0.021 & 40.0 \\
\hline $\mathrm{p} 4.4 . \mathrm{m}$ & 919 & 420 & 0.12 & 356 & 0.023 & 42.5 \\
\hline $\mathrm{p} 4.4 . \mathrm{n}$ & 977 & 420 & 0.114 & 370 & 0.022 & 45.0 \\
\hline p4.4.o & 1061 & 451 & 0.12 & 392 & 0.026 & 47.5 \\
\hline p4.4.p & 1124 & 458 & 0.123 & 380 & 0.116 & 50.0 \\
\hline p4.4.q & 1161 & 486 & 0.147 & 382 & 0.021 & 52.5 \\
\hline
\end{tabular}

Table 15. The computational results for data set 5 with $\mathrm{m}=2$

\begin{tabular}{|c|c|c|c|c|c|c|}
\hline Problem & Best-known solution & Best-obtained solution & Time (Second) & Best-obtained solution & Time (Second) & $T_{\max }$ \\
\hline$\overline{\mathrm{p} 5.2 . \mathrm{a}}$ & 0 & 0 & 0.035 & 0 & 0.009 & 2.5 \\
\hline p5 $5.2 . b$ & 20 & 20 & 0.011 & 20 & 0.011 & 5.0 \\
\hline p5.2.c & 50 & 50 & 0.02 & 40 & 0.01 & 7.5 \\
\hline p5.2.d & 80 & 80 & 0.021 & 70 & 0.013 & 10.0 \\
\hline p5.2.e & 180 & 170 & 0.035 & 135 & 0.025 & 12.5 \\
\hline p5.2.f & 240 & 175 & 0.023 & 145 & 0.066 & 15.0 \\
\hline p5.2.g & 320 & 205 & 0.137 & 190 & 0.015 & 17.5 \\
\hline p5.2.h & 410 & 270 & 0.069 & 220 & 0.014 & 20.0 \\
\hline p5 5.2 .1 & 480 & 320 & 0.041 & 275 & 0.025 & 22.5 \\
\hline p5.2.j & 580 & 350 & 0.071 & 270 & 0.011 & 25.0 \\
\hline $\mathrm{p} 5.2 . \mathrm{k}$ & 670 & 375 & 0.083 & 375 & 0.04 & 27.5 \\
\hline p5 5.2 .1 & 800 & 435 & 0.067 & 390 & 0.032 & 30.0 \\
\hline p5.2.m & 860 & 430 & 0.072 & 375 & 0.024 & 32.5 \\
\hline p5.2.n & 925 & 460 & 0.066 & 425 & 0.031 & 35.0 \\
\hline p5.2.o & 1020 & 470 & 0.072 & 410 & 0.026 & 37.5 \\
\hline p5.2.p & 1150 & 480 & 0.202 & 470 & 0.034 & 40.0 \\
\hline p5.2.q & 1195 & 550 & 0.094 & 550 & 0.045 & 42.5 \\
\hline p5.2.r & 1260 & 575 & 0.089 & 450 & 0.041 & 45.0 \\
\hline p5 $5.2 . \mathrm{s}$ & 1340 & 570 & 0.088 & 510 & 0.046 & 47.5 \\
\hline p5.2.t & 1400 & 580 & 0.094 & 550 & 0.043 & 50.0 \\
\hline p5.2.u & 1460 & 610 & 0.111 & 570 & 0.06 & 52.5 \\
\hline p5 $5.2 . \mathrm{v}$ & 1505 & 640 & 0.118 & 575 & 0.044 & 55.0 \\
\hline p5.2.w & 1565 & 660 & 0.115 & 560 & 0.046 & 57.5 \\
\hline p5.2.x & 1610 & 660 & 0.117 & 585 & 0.054 & 60.0 \\
\hline p5.2.y & 1645 & 710 & 0.116 & 570 & 0.035 & 62.5 \\
\hline p5.2.z & 1680 & 730 & 0.123 & 610 & 0.046 & 65.0 \\
\hline
\end{tabular}


Manal Zettam / Journal of Computer Science 2017, 13 (9): 440.451 DOI: $10.3844 /$ jessp.2017.440.451

Table 16. The computational results for data set 5 with $\mathrm{m}=3$

\begin{tabular}{|c|c|c|c|c|c|c|}
\hline Problem & $\begin{array}{l}\text { Best-known } \\
\text { solution }\end{array}$ & $\begin{array}{l}\text { Best-obtained } \\
\text { solution }\end{array}$ & $\begin{array}{l}\text { Time } \\
\text { (Second) }\end{array}$ & $\begin{array}{l}\text { Best-obtained } \\
\text { solution }\end{array}$ & $\begin{array}{l}\text { Time } \\
\text { (Second) }\end{array}$ & $T_{\max }$ \\
\hline p5.3.a & 0 & 0 & 0.005 & 0 & 0.009 & 1.7 \\
\hline p5 $5.3 . b$ & 15 & 15 & 0.01 & 15 & 0.008 & 3.3 \\
\hline p5.3.c & 20 & 20 & 0.02 & 20 & 0.01 & 5.0 \\
\hline p5.3.d & 60 & 60 & 0.02 & 60 & 0.01 & 6.7 \\
\hline p5.3.e & 95 & 95 & 0.02 & 95 & 0.016 & 8.3 \\
\hline p5.3.f & 110 & 110 & 0.03 & 110 & 0.01 & 10.0 \\
\hline p5.3.g & 185 & 150 & 0.02 & 140 & 0.01 & 11.7 \\
\hline p5.3.h & 260 & 210 & 0.06 & 185 & 0.03 & 13.3 \\
\hline p5.3.i & 335 & 240 & 0.04 & 220 & 0.07 & 15.0 \\
\hline p5.3.j & 470 & 285 & 0.03 & 275 & 0.02 & 16.7 \\
\hline $\mathrm{p} 5.3 . \mathrm{k}$ & 495 & 335 & 0.08 & 305 & 0.01 & 18.3 \\
\hline p5.3.1 & 595 & 385 & 0.06 & 335 & 0.02 & 20.0 \\
\hline p5.3.m & 650 & 425 & 0.06 & 390 & 0.02 & 21.7 \\
\hline p5.3.n & 755 & 455 & 0.06 & 410 & 0.02 & 23.3 \\
\hline p5.3.o & 870 & 510 & 0.081 & 345 & 0.03 & 25.0 \\
\hline p5.3.p & 990 & 535 & 0.08 & 475 & 0.02 & 26.7 \\
\hline p5.3.q & 1070 & 510 & 0.07 & 530 & 0.07 & 28.3 \\
\hline p5.3.r & 1125 & 595 & 0.08 & 570 & 0.05 & 30.0 \\
\hline p5.3.s & 1190 & 610 & 0.1 & 610 & 0.05 & 31.7 \\
\hline p5 $5.3 . t$ & 1260 & 595 & 0.1 & 515 & 0.024 & 33.3 \\
\hline p5.3.u & 1345 & 630 & 0.09 & 560 & 0.09 & 35.0 \\
\hline p5.3.v & 1425 & 695 & 0.09 & 530 & 0.03 & 36.7 \\
\hline p5.3.w & 1485 & 645 & 0.09 & 595 & 0.065 & 38.3 \\
\hline p5.3.x & 1555 & 645 & 0.08 & 660 & 0.044 & 40.0 \\
\hline p5.3.y & 1595 & 735 & 0.112 & 690 & 0.04 & 41.7 \\
\hline p5.3.z & 1635 & 755 & 0.13 & 695 & 0.052 & 43.3 \\
\hline
\end{tabular}

Table 17. The computational results for data set 5 with $\mathrm{m}=4$

\begin{tabular}{|c|c|c|c|c|c|c|}
\hline Problem & $\begin{array}{l}\text { Best-known } \\
\text { solution }\end{array}$ & $\begin{array}{l}\text { Best-obtained } \\
\text { solution }\end{array}$ & $\begin{array}{l}\text { Time } \\
\text { (Second) }\end{array}$ & $\begin{array}{l}\text { Best-obtained } \\
\text { solution }\end{array}$ & $\begin{array}{l}\text { Time } \\
\text { (Second) }\end{array}$ & $T_{\max }$ \\
\hline p5.4.a & 0 & 0 & 0.01 & 0 & 0.012 & 1.2 \\
\hline p5.4.b & 0 & 0 & 0.008 & 0 & 0.06 & 2.5 \\
\hline p5.4.c & 20 & 20 & 0.02 & 20 & 0.001 & 3.8 \\
\hline p5.4.d & 20 & 20 & 0.02 & 20 & 0.01 & 5.0 \\
\hline p5.4.e & 20 & 20 & 0.019 & 20 & 0.01 & 6.2 \\
\hline p5.4.f & 80 & 80 & 0.02 & 80 & 0.01 & 7.5 \\
\hline p5.4.g & 140 & 140 & 0.035 & 125 & 0.01 & 8.8 \\
\hline p5.4.h & 140 & 140 & 0.03 & 140 & 0.01 & 10.0 \\
\hline p5.4.i & 240 & 200 & 0.24 & 185 & 0.021 & 11.2 \\
\hline p5.4.j & 340 & 230 & 0.05 & 210 & 0.06 & 12.5 \\
\hline p5.4.k & 340 & 285 & 0.06 & 270 & 0.02 & 13.8 \\
\hline p5.4.1 & 430 & 310 & 0.05 & 305 & 0.024 & 15.0 \\
\hline p5.4.m & 555 & 360 & 0.059 & 345 & 0.02 & 16.2 \\
\hline p5.4.n & 620 & 405 & 0.05 & 375 & 0.017 & 17.5 \\
\hline p5.4.o & 690 & 435 & 0.06 & 405 & 0.032 & 18.8 \\
\hline p5.4.p & 765 & 490 & 0.076 & 435 & 0.02 & 20.0 \\
\hline p5.4.q & 860 & 500 & 0.07 & 470 & 0.03 & 21.2 \\
\hline p5.4.r & 960 & 540 & 0.08 & 505 & 0.032 & 22.5 \\
\hline p5.4.s & 1030 & 580 & 0.08 & 535 & 0.03 & 23.8 \\
\hline p5.4.t & 1160 & 595 & 0.08 & 560 & 0.02 & 25.0 \\
\hline $\mathrm{p} 5.4 . \mathrm{u}$ & 1300 & 660 & 0.091 & 590 & 0.041 & 26.2 \\
\hline p5.4.v & 1320 & 705 & 0.09 & 635 & 0.041 & 27.5 \\
\hline p5.4.w & 1390 & 650 & 0.111 & 650 & 0.06 & 28.8 \\
\hline p5.4.x & 1450 & 675 & 0.1 & 670 & 0.05 & 30.0 \\
\hline p5.4.y & 1520 & 740 & 0.12 & 630 & 0.03 & 31.2 \\
\hline p5.4.z & 1620 & 740 & 0.09 & 640 & 0.04 & 32.5 \\
\hline
\end{tabular}


Manal Zettam / Journal of Computer Science 2017, 13 (9): 440.451 DOI: $10.3844 /$ jessp.2017.440.451

Table 18. The computational results for data set 6 with $\mathrm{m}=2$

\begin{tabular}{|c|c|c|c|c|c|c|}
\hline Problem & Best-known solution & Best-obtained solution & Time(Second) & Best-obtained solution & Time(Second) & $T_{\max }$ \\
\hline p6.2.a & 0 & 0 & 0.01 & 0 & 0.009 & 7.5 \\
\hline p6.2.b & 0 & 0 & 0.016 & 0 & 0.01 & 10.0 \\
\hline p6.2.c & 0 & 0 & 0.014 & 0 & 0.009 & 12.5 \\
\hline p6.2.d & 192 & 168 & 0.02 & 156 & 0.02 & 15.0 \\
\hline p6.2.e & 360 & 264 & 0.03 & 204 & 0.039 & 17.5 \\
\hline p6.2.f & 588 & 348 & 0.04 & 288 & 0.01 & 20.0 \\
\hline p6.2.g & 660 & 432 & 0.041 & 330 & 0.024 & 22.5 \\
\hline p6.2.h & 780 & 402 & 0.09 & 366 & 0.025 & 25.0 \\
\hline p6.2.i & 888 & 468 & 0.059 & 354 & 0.021 & 27.5 \\
\hline p6.2.j & 948 & 474 & 0.065 & 402 & 0.05 & 30.0 \\
\hline p6.2.k & 1032 & 516 & 0.067 & 414 & 0.031 & 32.5 \\
\hline p6.2.1 & 1116 & 528 & 0.069 & 432 & 0.03 & 35.0 \\
\hline p6.2.m & 1188 & 546 & 0.07 & 450 & 0.033 & 37.5 \\
\hline p6.2.n & 1260 & 606 & 0.084 & 480 & 0.09 & 40.0 \\
\hline
\end{tabular}

Table 19. The computational results for data set 6 with $\mathrm{m}=3$

\begin{tabular}{|c|c|c|c|c|c|c|}
\hline Problem & Best-known solution & Best-obtained solution & Time (Second) & Best-obtained solution & Time (Second) & $T_{\max }$ \\
\hline p6.3.a & 0 & 0 & 0.011 & 0 & 0.009 & 5.0 \\
\hline p6.3.b & 0 & 0 & 0.01 & 0 & 0.009 & 6.7 \\
\hline p6.3.c & 0 & 0 & 0.05 & 0 & 0.01 & 8.3 \\
\hline p6.3.d & 0 & 0 & 0.02 & 0 & 0.02 & 10.0 \\
\hline p6.3.e & 0 & 0 & 0.02 & 0 & 0.01 & 11.7 \\
\hline p6.3.f & 0 & 0 & 0.03 & 0 & 0.01 & 13.3 \\
\hline p6.3.g & 282 & 234 & 0.044 & 216 & 0.042 & 15.0 \\
\hline p6.3.h & 444 & 336 & 0.05 & 288 & 0.01 & 16.7 \\
\hline p6.3.i & 642 & 420 & 0.05 & 336 & 0.02 & 18.3 \\
\hline p6.3.j & 828 & 468 & 0.098 & 420 & 0.03 & 20.0 \\
\hline p6.3.k & 894 & 534 & 0.06 & 444 & 0.03 & 21.7 \\
\hline p6.3.1 & 1002 & 534 & 0.07 & 486 & 0.036 & 23.3 \\
\hline $\mathrm{p} 6.3 . \mathrm{m}$ & 1080 & 636 & 0.07 & 546 & 0.045 & 25.0 \\
\hline p6.3.n & 1170 & 654 & 0.071 & 528 & 0.045 & 26.7 \\
\hline
\end{tabular}

Table 20. The computational results for data set 6 with $\mathrm{m}=4$

\begin{tabular}{|c|c|c|c|c|c|c|}
\hline Problem & Best-known solution & Best-obtained solution & Time (Second) & Best-obtained solution & Time (Second) & $T_{\max }$ \\
\hline p6.4.a & 0 & 0 & 0.01 & 0 & 0.01 & 3.8 \\
\hline p6.4.b & 0 & 0 & 0.014 & 0 & 0.008 & 5.0 \\
\hline p6.4.c & 0 & 0 & 0.016 & 0 & 0.01 & 6.2 \\
\hline p6.4.d & 0 & 0 & 0.018 & 0 & 0.008 & 7.5 \\
\hline p6.4.e & 0 & 0 & 0.023 & 0 & 0.005 & 8.8 \\
\hline p6.4.f & 0 & 0 & 0.025 & 0 & 0.009 & 10.0 \\
\hline p6.4.g & 0 & 0 & 0.025 & 0 & 0.007 & 11.2 \\
\hline p6.4.h & 0 & 0 & 0.03 & 0 & 0.009 & 12.5 \\
\hline p6.4.i & 0 & 0 & 0.032 & 0 & 0.006 & 13.8 \\
\hline p6.4.j & 366 & 294 & 0.047 & 264 & 0.015 & 15.0 \\
\hline p6.4.k & 528 & 408 & 0.058 & 312 & 0.018 & 16.2 \\
\hline p6.4.1 & 696 & 450 & 0.058 & 426 & 0.02 & 17.5 \\
\hline p6.4.m & 912 & 552 & 0.086 & 480 & 0.031 & 18.8 \\
\hline p6.4.n & 1068 & 564 & 0.069 & 498 & 0.028 & 20.0 \\
\hline
\end{tabular}

Table 3 contains the results obtained for data set 1 with 2 tours. Six best-known solutions are obtained for a population size equal to $10 \times T_{\max }$, while only four bestknown solutions are obtained for a population size equal to 10 . The results obtained for data set 1 with 3 tours are contained in Table 4. Eight best-known solutions are obtained for population size equal to $10 \times T_{\max }$, while seven best-known solutions are obtained for a population size equal to 10 . The reminder tables contain the results of the rest sets. The obtained results by population size equal to $10 \times T_{\max }$ are better than those obtained by a population size equal to 10 .

The obtained results prove the efficiency of our heuristic. Indeed, a great number of solutions is near to the best-known solutions. Other obtained solutions reach the best-known solution in minor computational time. Those results represent a promising start for populationbased metaheuristics. 
Manal Zettam / Journal of Computer Science 2017, 13 (9): 440.451 DOI: $10.3844 /$ jessp.2017.440.451

Table 21. The computational results for data set 7 with $\mathrm{m}=2$

\begin{tabular}{lllllll}
\hline Problem & Best-known solution & Best-obtained solution & Time (Second) & Best-obtained solution & Time (Second) & $T_{\max }$ \\
\hline p7.2.a & 30 & 30 & 0.023 & 30 & 0.008 & 10.0 \\
p7.2.b & 64 & 64 & 0.008 & 64 & 0.008 & 20.0 \\
p7.2.c & 101 & 101 & 0.059 & 101 & 0.013 & 30.0 \\
p7.2.d & 190 & 156 & 0.083 & 131 & 0.074 & 40.0 \\
p7.2.e & 290 & 202 & 0.093 & 158 & 0.009 & 50.0 \\
p7.2.f & 387 & 255 & 0.105 & 191 & 0.021 & 70.0 \\
p7.2.g & 459 & 277 & 0.113 & 229 & 0.014 & 80.0 \\
p7.2.h & 521 & 269 & 0.127 & 232 & 0.019 & 90.0 \\
p7.2.i & 580 & 307 & 0.141 & 239 & 0.027 & 100.0 \\
p7.2.j & 646 & 325 & 0.15 & 257 & 0.073 & 110.0 \\
p7.2.k & 705 & 329 & 0.184 & 267 & 0.018 & 120.0 \\
p7.2.1 & 767 & 348 & 0.173 & 289 & 0.028 & 130.0 \\
p7.2.m & 827 & 348 & 0.207 & 312 & 0.01 & 140.0 \\
p7.2.n & 888 & 389 & 0.212 & 257 & 0.02 & 150.0 \\
p7.2.0 & 945 & 401 & 0.222 & 321 & 0.02 & 160.0 \\
p7.2.p & 1002 & 386 & 0.241 & 312 & 0.03 & 170.0 \\
p7.2.q & 1044 & 404 & 0.24 & 333 & 0.022 & 180.0 \\
p7.2.r & 1094 & 414 & 0.251 & 393 & 0.02 & 190.0 \\
p7.2.s & 1136 & 429 & 0.327 & 416 & 0.023 & 200.0 \\
p7.2.t & 1179 & 441 & 0.301 & 383 & & 0.0 \\
\hline
\end{tabular}

Table 22. The computational results for data set 7 with $\mathrm{m}=3$

\begin{tabular}{|c|c|c|c|c|c|c|}
\hline Problem & Best-known solution & Best-obtained solution & Time (Second) & Best-Obtained Solution & Time (Second) & $T_{\max }$ \\
\hline p7.3.a & 0 & 0 & 0.02 & 0 & 0.004 & 6.7 \\
\hline p7.3.b & 46 & 46 & 0.03 & 46 & 0.05 & 13.3 \\
\hline p7.3.c & 79 & 79 & 0.05 & 79 & 0.01 & 20.0 \\
\hline p7.3.d & 117 & 117 & 0.07 & 117 & 0.03 & 26.7 \\
\hline p7.3.e & 175 & 175 & 0.23 & 148 & 0.009 & 33.3 \\
\hline p7.3.f & 247 & 202 & 0.11 & 186 & 0.02 & 40.0 \\
\hline p7.3.g & 344 & 253 & 0.1 & 214 & 0.13 & 46.7 \\
\hline p7.3.h & 425 & 296 & 0.11 & 252 & 0.009 & 53.3 \\
\hline p7.3.i & 487 & 311 & 0.13 & 269 & 0.01 & 60.0 \\
\hline p7.3.j & 564 & 331 & 0.142 & 293 & 0.02 & 66.7 \\
\hline p7.3.k & 633 & 350 & 0.17 & 253 & 0.02 & 73.3 \\
\hline p7.3.1 & 684 & 357 & 0.16 & 305 & 0.02 & 80.0 \\
\hline p7.3.m & 762 & 388 & 0.18 & 304 & 0.025 & 86.7 \\
\hline p7.3.n & 820 & 389 & 0.212 & 322 & 0.021 & 93.3 \\
\hline p7.3.o & 874 & 403 & 0.21 & 346 & 0.02 & 100.0 \\
\hline p7.3.p & 929 & 417 & 0.24 & 335 & 0.027 & 106.7 \\
\hline p7.3.q & 987 & 475 & 0.241 & 388 & 0.018 & 113.3 \\
\hline p7.3.r & 1026 & 450 & 0.25 & 389 & 0.02 & 120.0 \\
\hline p7.3.s & 1081 & 446 & 0.26 & 382 & 0.02 & 126.7 \\
\hline p7.3.t & 1120 & 473 & 0.281 & 401 & 0.022 & 133.3 \\
\hline
\end{tabular}

Table 23. The computational results for data set 7 with $\mathrm{m}=4$

\begin{tabular}{|c|c|c|c|c|c|c|}
\hline Problem & Best-known solution & Best-obtained solution & Time (Second) & Best-obtained solution & Time (Second) & $T_{\max }$ \\
\hline p7.4.a & 0 & 0 & 0.02 & 0 & 0.01 & 5.0 \\
\hline p7.4.b & 30 & 30 & 0.03 & 30 & 0.012 & 10.0 \\
\hline p7.4.c & 46 & 46 & 0.05 & 46 & 0.01 & 15.0 \\
\hline p7.4.d & 79 & 79 & 0.07 & 79 & 0.01 & 20.0 \\
\hline p7.4.e & 123 & 123 & 0.07 & 123 & 0.06 & 25.0 \\
\hline p7.4.f & 164 & 164 & 0.08 & 155 & 0.01 & 30.0 \\
\hline p7.4.g & 217 & 203 & 0.11 & 195 & 0.01 & 35.0 \\
\hline p7.4.h & 285 & 247 & 0.121 & 222 & 0.02 & 40.0 \\
\hline p7.4.i & 366 & 273 & 0.122 & 272 & 0.02 & 45.0 \\
\hline p7.4.j & 462 & 326 & 0.15 & 287 & 0.028 & 50.0 \\
\hline p7.4.k & 520 & 340 & 0.16 & 339 & 0.026 & 55.0 \\
\hline p7.4.1 & 590 & 376 & 0.16 & 351 & 0.02 & 60.0 \\
\hline $\mathrm{p} 7.4 . \mathrm{m}$ & 646 & 399 & 0.194 & 343 & 0.02 & 65.0 \\
\hline p7.4.n & 730 & 422 & 0.226 & 370 & 0.021 & 70.0 \\
\hline p7.4.o & 781 & 436 & 0.357 & 401 & 0.034 & 75.0 \\
\hline p7.4.p & 846 & 456 & 0.223 & 398 & 0.026 & 80.0 \\
\hline p7.4.q & 909 & 457 & 0.232 & 409 & 0.023 & 85.0 \\
\hline p7.4.r & 970 & 490 & 0.254 & 388 & 0.024 & 90.0 \\
\hline p7.4.s & 1022 & 498 & 0.254 & 399 & 0.028 & 95.0 \\
\hline p7.4.t & 1077 & 506 & 0.271 & 449 & 0.03 & 100.0 \\
\hline
\end{tabular}




\section{Conclusion}

This paper, introduces a randomized population constructive that generates the initial population for the team orienteering problem. The obtained results promote the integration of the heuristic to built initial population for population-based metaheuristics. The use of permutations for the proposed heuristic avoids infeasible solutions. Moreover, dealing with permutations during the search process would facilitate the application of operators and avoid infeasible solutions. Indeed, for each permutation a unique solution is associated by applying only the extern loop of the proposed heuristic. In this study, the best solution of the population is enhanced by a local search using the swap and the add/drop operators. This component allows finding better solutions.

\section{Ethics}

The author confirms that this manuscript has not been published elsewhere and that no ethical issues are involved.

\section{References}

Archetti, C., A. Hertz and M.G. Speranza, 2007. Metaheuristics for the team orienteering problem. J. Heurist., 13: 49-76. DOI: $10.1007 / \mathrm{s} 10732-006-9004-0$

Bouly, H., D.C. Dang and A. Moukrim, 2009. A memetic algorithm for the team orienteering problem. 4OR, 8: 49-70.

DOI: $10.1007 / \mathrm{s} 10288-008-0094-4$

Butt, S.E. and T.M. Cavalier, 1994. A heuristic for the multiple tour maximum collection problem. Comput. Oper. Res., 21: 101-111. DOI: 10.1016/0305-0548(94)90065-5

Chao, I.M., B.L. Golden and E.A. Wasil, 1996. The team orienteering problem. Eur. J. Operat. Res., 88: 464-474. DOI: 10.1016/0377-2217(94)00289-4

Golden, B.L., L. Levy and R. Vohra, 1987. The orienteering problem. Nav. Res. Logist., 34: 307-318. DOI: $\quad 10.1002 / 1520-6750(198706) 34: 3<307:: A I D-$ NAV3220340302>3.0.CO;2-D
Ke, L., C. Archetti and Z. Feng, 2008. Ants can solve the team orienteering problem. Comput. Ind. Eng., 54: 648-665. DOI: 10.1016/j.cie.2007.10.001

Kim, B.I., H. Li and A.L. Johnson, 2013. An augmented large neighborhood search method for solving the team orienteering problem. Expert Syst. Applic., 40: 3065-3072.

DOI: $10.1016 /$ j.eswa.2012.12.022

Lin, S.W., 2013. Solving the team orienteering problem using effective multi-start simulated annealing. Applied Soft Comput. 13: 1064-1073.

DOI: 10.1016/j.asoc.2012.09.022

Souffriau, W., P. Vansteenwegen, J. Vertommen, G.V. Berghe and D.V. Oudheusden, 2008. A personalized tourist trip design algorithm for mobile tourist guides. Applied Artif. Intell., 22: 964-985. DOI: $10.1080 / 08839510802379626$

Tang, H. and E. Miller-Hooks, 2005. A TABU search heuristic for the team orienteering problem. Comput. Operat. Res., 32: 1379-1407.

DOI: $10.1016 /$ j.cor.2003.11.008

Thomadsen, T. and T. Stidsen, 2003. The quadratic selective travelling salesman problem. Informatics and Mathematical Modelling, Technical University of Denmark, DTU.

Tsiligirides, T., 1984. Heuristic methods applied to orienteering. J. Operat. Res. Society, 35: 797-809. DOI: 10.1057 /jors. 1984.162

Vansteenwegen, P., W. Souffriau, G.V. Berghe and D.V. Oudheusden, 2009. A guided local search metaheuristic for the team orienteering problem. Eur. J. Operat. Res., $196: 118-127$. DOI: 10.1016/j.ejor.2008.02.037

Vansteenwegen, P., W. Souffriau and D.V. Oudheusden, 2011. The orienteering problem: A survey. Eur. J. Operat. Res., $209: 1-10$. DOI: 10.1016/j.ejor.2010.03.045

Zettam, M. and B. Elbenani, 2016. A novel randomized heuristic for the team orienteering problem. Proceedings of the 3rd International Conference on Logistics Operations Management, May 23-25, IEEE Xplore Press, pp: 1-6.

DOI: $10.1109 /$ GOL.2016.7731671 